\title{
Trade Studies for a Manned High-Power Nuclear Electric Propulsion Vehicle
}

\author{
Michael SanSoucie ${ }^{*}$ and Patrick V. Hull* \\ Universities Space Research Association, NASA Marshall Space Flight Center, Huntsville, Alabama, 35812 \\ Ryan W. Irwin \\ School of Aeronautics and Astronautics, Purdue University, West Lafayette, IN 47907 \\ and \\ Michael L. Tinker ${ }^{\dagger}$ and Bruce W. Patton ${ }^{\ddagger}$ \\ NASA Marshall Space Flight Center, Huntsville, Alabama, 35812
}

\begin{abstract}
Nuclear electric propulsion (NEP) vehicles will be needed for future manned missions to Mars and beyond. Candidate vehicles must be identified through trade studies for further detailed design from a large array of possibilities. Genetic algorithms have proven their utility in conceptual design studies by effectively searching a large design space to pinpoint unique optimal designs. This research combines analysis codes for NEP subsystems with genetic algorithm-based optimization. Trade studies for a NEP reference mission to the asteroids were conducted to identify important trends, and to determine the effects of various technologies and subsystems on vehicle performance. It was found that the electric thruster type and thruster performance have a major impact on the achievable system performance, and that significant effort in thruster research and development is merited.
\end{abstract}

$\begin{array}{ll} & \\ A U & =\text { astronomical units } \\ L 1 & =\text { Lagrange point } \\ M P D & =\text { magnetoplasmadynamic } \\ N E P & =\text { nuclear electric propulsion } \\ N E V O T & =\text { nuclear electric vehicle optimization toolset } \\ P M A D & =\text { power management and distribution } \\ p & =\text { penalty ratio } \\ S R P S & =\text { space reactor power system } \\ x_{\text {design }} & =\text { calculated value of vehicle or mission parameter } \\ x_{\text {constraint }} & =\text { target or constraint value of vehicle or mission parameter }\end{array}$

\section{Introduction}

$\mathrm{T}$ HE announcement of the Vision for Space Exploration has resulted in renewed focus on human exploration of Mars and other distant locations. Nuclear electric propulsion (NEP) has been identified as a front runner for these missions that necessitate short trip times to minimize crew cosmic radiation exposure. While numerous manned chemical propulsion vehicles have been designed in the past, there is considerably less experience for nuclear electric propulsion vehicles. In addition to traditional aerospace knowledge areas, competent NEP design requires knowledge of nuclear reactors and electric thrusters as well as conversion, management, and distribution of thermal and electrical energy. A thorough search of the design possibilities for these vehicles is needed for a proper assessment of mission viability and identification of feasible low-mass candidates for future designs. Genetic

\footnotetext{
* Research Associate, Spacecraft and Vehicle Systems Department, EV11.

${ }^{+}$Aerospace Technologist, Aerospace Flight Systems; Spacecraft and Vehicle Systems Dept., EV11; Assoc. Fellow AIAA.

$\ddagger$ Aerospace Technologist, Electric Propulsion; Propulsion Research Center, XD20.
} 
algorithms have demonstrated an ability to find global optima over large search spaces such as this. Furthermore, genetic algorithms are suited for preliminary design problems because they can be used with both continuous and discrete design variables and can find areas of optimal designs for later detailed design analysis. This research aims at finding candidate nuclear electric vehicle designs for a manned mission to the asteroid belt.

\section{Nuclear Electric Propulsion}

Nuclear electric propulsion involves using a nuclear energy source (such as a fission reactor) to create a large amount of heat that can be converted into electric power for use by electric thrusters. A basic NEP vehicle system layout is shown in Figure 1. A manned mission to another planet would require thruster power greater than one megawatt, perhaps as much as 20 megawatis. These high power ieveis coupied with the smaii avaiiabie reactor power conversion efficiencies create large amounts of waste heat that must be dissipated by radiators. Currently, heat pipe radiators are considered the best option because the spacecraft can survive the loss of several heat pipes with only small loss in rejection ability, as demonstrated by Smith ${ }^{1}$.

Radiation created by the reactor requires a shadow shield to protect the crew habitat and other subsystem components. High shield densities require a large separation distance between the habitat and the reactor, which is accomplished by a truss. The length of the truss determines the size and shape of the radiation shielding. Increasing the separation distance results in thinner shields and smaller shadow angles, thereby decreasing the shield volume. Additionally, the truss must be adequately designed to withstand static and dynamic loads.

There are many types of electric propulsion systems under consideration for NEP missions, including ion, Hall, and magnetoplasmadynamic (MPD) thrusters. These thrusters provide tradeoffs between specific impulse and high power capability. For example, ion thrusters have high specific impulse, but several clusters of them are required to supply high jet power. This leads to tradeoffs in propellant efficiency and thruster mass.

\section{Reference Mission To The Asteroids}

The reference manned mission was selected to deliver a payload from the earth-moon L1 point to a destination 4 $\mathrm{AU}$ from the sun, to loiter there for 30 days, and to return the vehicle to the earth-moon L1 point. This mission is deliberately generic but demanding enough to demonstrate NEVOT capabilities and to allow an assessment of NEP technologies. The location at $4 \mathrm{AU}$ places the destination in the Hilda asteroid group, at the inner edge of the outermost Kirkwood gap (Figure 2). The target trip time for the reference mission is 4 years (1470 days), which the nuclear electric vehicle must accomplish to be competitive with chemical system trip times. The 1470 day mission corresponds to the Hohmann transfer time that would be used by a chemical system with the 30 day loiter added. Departure date is not specified in this analysis. 


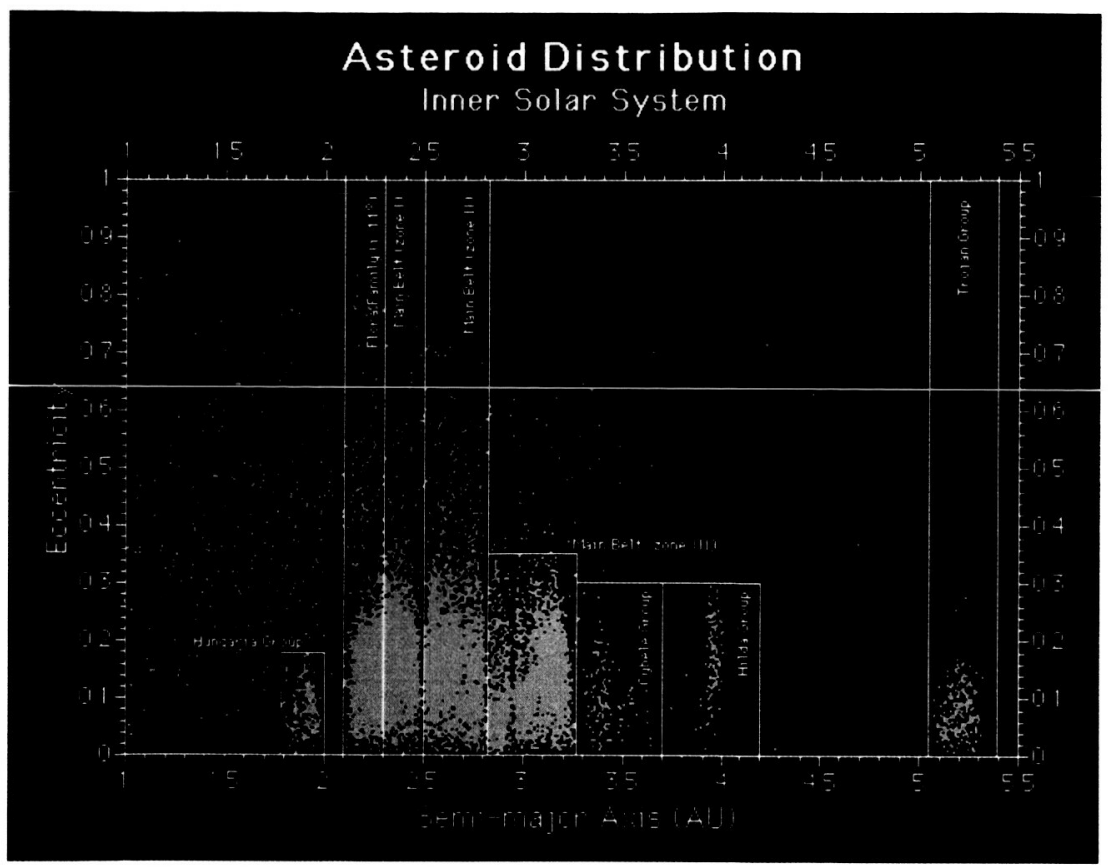

Figure 2. Reference mission destination to $4 \mathrm{AU}$ in asteroid belt.

The payload will be a human habitat accommodating a crew of three for the specified trip time. Associated guidelines are:

- Habitat will be assumed to be one cylinder

- Airlock/docking interface at one end of cylinder

- Artificial gravity of $1 \mathrm{~g}$ will be provided at airlock end via vehicle rotation about its main axis

- Habitat will be attached to vehicle central truss (long axis) at end opposite airlock

- Habitat diameter is a variable in the simulations, but within reasonable range $(5.0 \mathrm{~m}$ is consistent with anticipated available shroud diameters)

- Radiation shelter for solar flare events

- ECLSS closed for air and water only

- Waste products expended continuously throughout the mission

- Crew systems to include spacesuits interchangeable between crewmembers, spares

- Two MMUs and associated masses for 12 translations, each of distance $200 \mathrm{~m}$ (roundtrip)

The required data as a function of trip time are:

- Habitat length

- Total mass and mass expenditure rate

- Center of mass and moments of inertia along cylinder axis and in two orthogonal directions. The last two axes will be aligned parallel and perpendicular to the plane of the vehicle radiators.

Trajectory analysis was performed in VariTOP resulting in an optimized electrical power for a fixed range of $\mathrm{I}_{\mathrm{sp}}$ values, a fixed range of trip times, and a single fixed power and propulsion system specific mass (alpha). The reference mission trajectory is shown in Figure 3. The traces in bold black indicate periods of thrust during the mission. Within the trajectory module each vehicle's total electrical power, the thruster $\mathrm{I}_{\mathrm{sp}}$, and total vehicle mass are used to perform a table look-up into the VariTOP data (Figure 4), which results in a corresponding estimate of trip time. To provide greater fidelity for future efforts, the VariTOP analysis should be expanded to include a greater range of $\mathrm{I}_{\mathrm{sp}}$ values, a greater range of trip times, and a greater range of alpha (ratio of vehicle dry mass to thruster electrical power) values. 


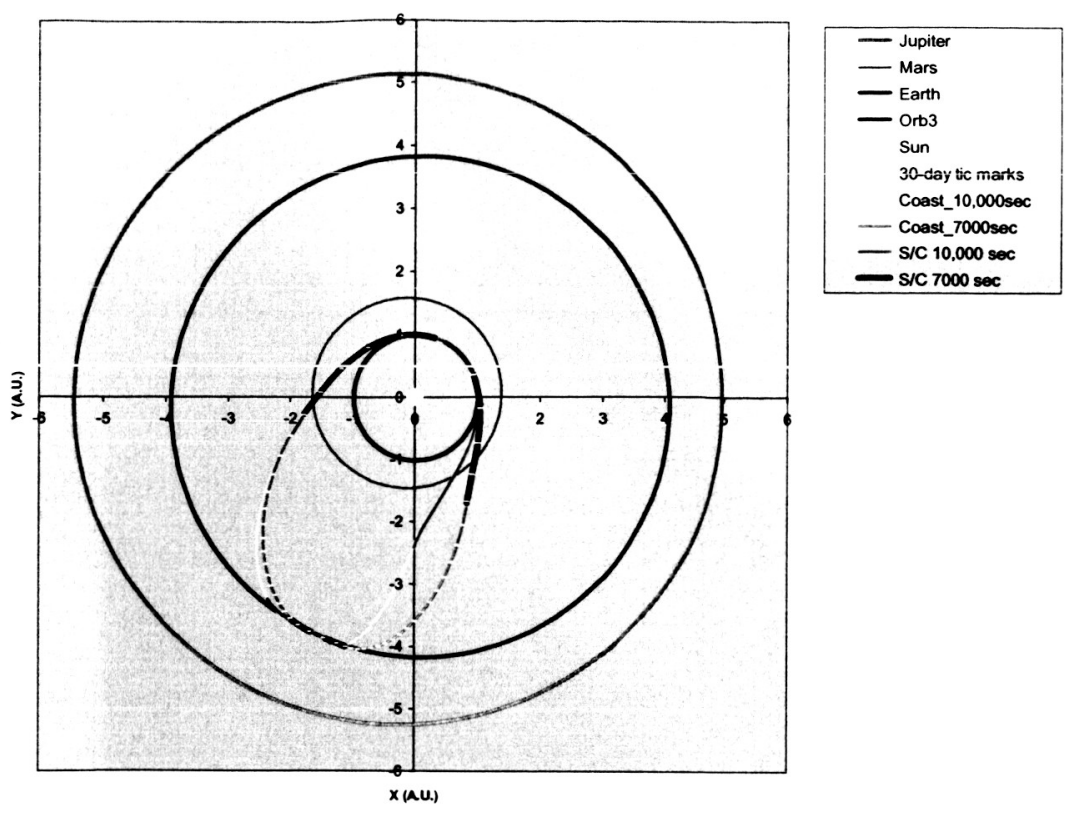

Figure 3. Trajectory for reference mission to $4 \mathrm{AU}$ in asteroid belt.

\section{EP TripTime vs P0}

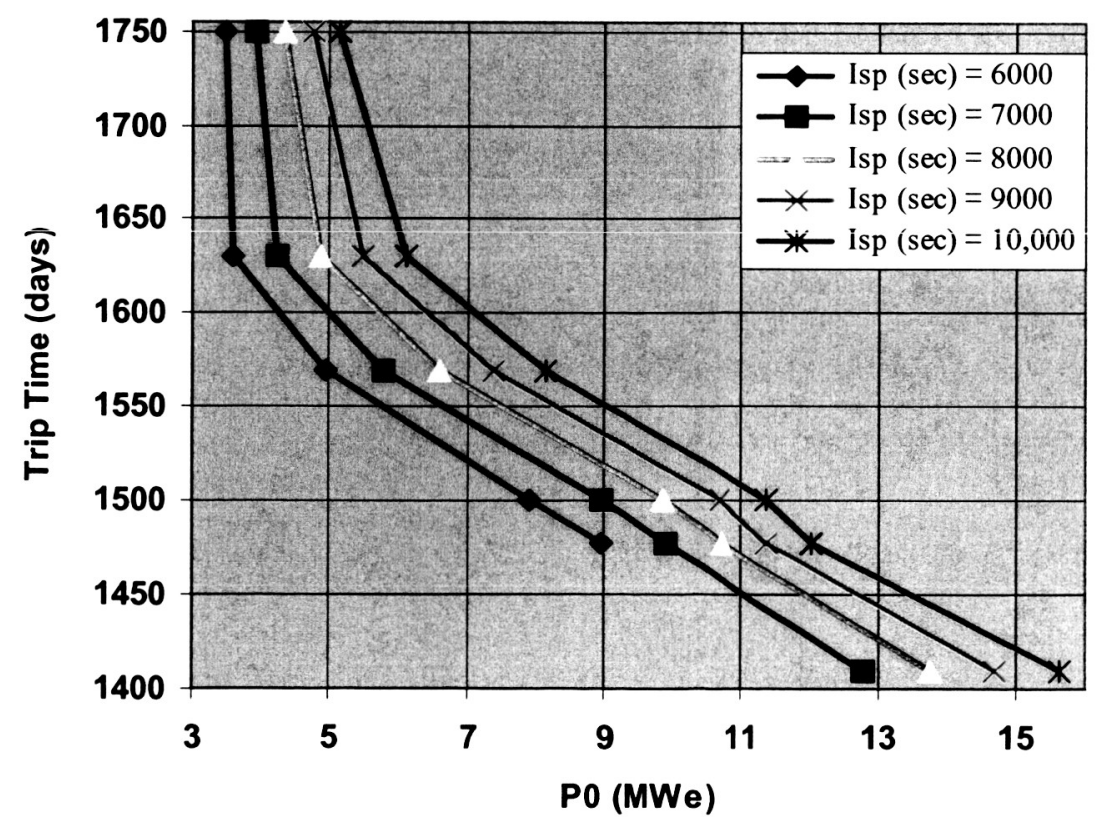

Figure 4. VariTOP trajectory data used for trip time estimates in NEVOT simulation. 


\section{Genetic Algorithms}

The optimization technique used in this research is a genetic programming approach. The traditional gradient search method and the employed genetic algorithm approach are discussed here. The traditional approach for optimizing design problems is the gradient based technique, which is typically quick and easy to implement. However, this optimization method is a local optimization method and it does not succumb to a thorough solution search of the multi-modal and non-convex search region. Many researchers are using this method for design problems including Ananthasuresh ${ }^{2}, \mathrm{Kota}^{3}$ and Parkinson ${ }^{4}$. A description of the genetic algorithm process follows.

Genetic algorithms are a global optimization technique ideal for problems where gradient information is unavailable, where many local optima exist, and where large search regions exist. As the name implies, genetic

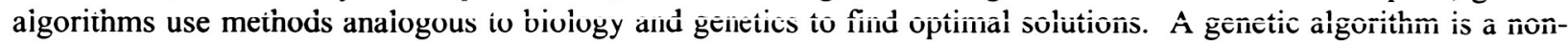
deterministic arbitrary guided search technique that covers the global region of the design space. Genetic algorithm searches are capable of managing both convex and non-convex objective functions. The genetic algorithm optimization method is defined by Parsons ${ }^{6}$ as "a mathematical approximation of Charles Darwin's theory on natural selection and the method of genetic information exchange in sexual reproduction."

A search of this nature classifies the potential solutions through a numerical description in the form of a coded number string called a chromosome that defines the design variables. The GA generates an initial population of designs (random or selected by the user) and defines a fitness value for each. The fitness value is defined by means of an objective function that the user supplies based on the problem of interest. When a population has been evaluated, the GA selects the solutions with the best fitness to undergo a genetic operation. The strings of these designs are replicated, crossed with one another, and/or mutated to create the next generation. Crossover occurs by swapping multiple portions of two parent designs to create offspring designs. The crossover points and mutated variables are determined randomly, and the rate at which they occur can be defined by the user. When the new population is filled, the process is repeated. Thus, the genetic algorithm uses a random, directed search to find global optima. The genetic operations allow for exploration of a large design space during simultaneous optimization.

Genetic algorithms can be combined with other search tools, thus providing solutions for final refinement by a specific optimization tool. They also have the ability to perform multi-objective optimization as required by a general design tool ${ }^{6,8}$. The application of genetic algorithms also poses some restrictions. In general, GA's have a comparatively high computational price because they are non-deterministic in nature and have a convergence performance that is complicated to foresee.

The genetic algorithm search used in this research starts with a family of randomly generated nuclear exploration vehicle solutions that meet some basic requirements; these solutions are analyzed and ranked according to a predefined fitness objective. The "most-fit" designs in the family of solutions are either passed along with their offspring to the next generation or are mated and only the offspring are passed on. The final outcome is a family of solutions that are clustered around the global minimum of the design region. Search procedures of this nature are computationally expensive, but the answers are by and large considered to be in the global optimal region.

For design problems there are often many constraints to consider. Penalty functions are used as a means to penalize the fitness of a solution for constraint violations. If the constraint is satisfied, then the penalty function value is zero. For instance, in the case of NEP vehicle mass optimization, the fitness function should be the sum of the system mass and any penalty functions for constraint violations. Generally, penalty functions are implemented by multiplying a scaling factor with a ratio based on the degree of the constraint violation. Determining an appropriate scaling factor involves some experimentation, as is discussed in later sections of this paper. 


\section{Nuclear Electric Vehicle Optimization Toolset (NEVOT)}

In order to combine the optimization power of a genetic algorithm with nuclear electric vehicle subsystem analyses, the Nuclear Electric Vehicle Optimization Toolset (NEVOT) was created ${ }^{9}$. NEVOT is a joint effort of Marshall Space Flight Center, Oak Ridge National Laboratories, and the Arnold Engineering Development Center. Figure 5 displays the general organization of NEVOT and its sublevel codes. There are seven analysis modules: Trajectory, Space Reactor Power System (SRPS), Power Management and Distribution (PMAD), Electric Propulsion (EP), Habitat (HAB), Truss, and Configuration (CONFIG). The input and output files for these modules are controlled in the simulation executive. This simulator combines all of the mass and performance data from the subsystem modules to calculate the system mass and assess any penalty functions for constraint violations. It is also

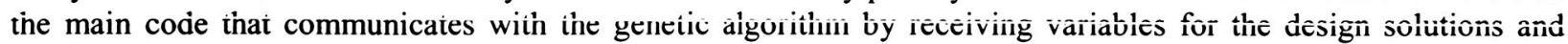
sending the corresponding fitness value back. The genetic algorithm used for the optimization is Sandia Laboratories' Design Analysis Kit for Optimization and Terascale Applications (DAKOTA) software package.

The current SRPS code is ALKASYS, a program developed at Oak Ridge National Laboratories for analysis of Rankinecycle space nuclear power systems. The PMAD code was developed in a collaborative effort between Rocketdyne and NASA's Glenn Research Center. The other analysis modules were developed by NEVOT project team members. Modularity of the NEVOT layout easily permits updates to these individual analysis modules. As codes are upgraded for increased accuracy and fidelity, they can be exchanged for previous versions of the codes in the computational architecture. For example, the original reactor code (RSMASS) was replaced by ALKASYS. RSMASS is a lower-fidelity reactor code that was used early in the project, but was removed after its analysis proved unreasonable over the mission power range.

Currently NEVOT allows the GA to operate on fifteen design variables: thruster jet power, thruster type (ion, Hall, or MPD), truss length, reactor type (boiling potassium or lithium cooled), condensing temperature, number of turbines, truss member outer diameter, truss member wall thickness, number of truss members, PMAD alternator operating frequency, thruster input voltage, transmission line length, propellant mass, specific impulse $\left(\mathrm{I}_{\mathrm{sp}}\right)$ and habitat diameter. Other values pertinent to the codes have been set as constants by engineering judgment. Several of the variables are used in more than one subsystem analysis code. Furthermore, the output and input power levels between connected subsystems must be consistent. Due to these dependencies, some subsystem codes must be run sequentially. The configuration module is run last since it uses the geometries generated by the other subsystems to size the shadow shield angle and diameters.

When a design is passed to the simulator, the subsystem modules are run for analysis of performance. A flowchart of how NEVOT is run is shown in Figure 6. The trajectory module uses the specific impulse and jet power to determine a mission trip time. This is later compared to a constraint target mission time chosen for crew health reasons. This trip time also is used in calculating the propellant mass required for the mission based on the jet power and specific impulse of the thrusters. This propellant mass requirement provides a constraint to check against 
the design propellant mass. The EP code is then run to obtain tank and thruster masses as well as the thrust force and thruster efficiency needed to calculate the PMAD output power. Then the PMAD module is run to obtain the mass and efficiency needed to calculate the SRPS output power. At this point, the reactor and power conversion subsystems are analyzed. This analysis yields SRPS and radiator masses as well as the required neutron and gamma shield thicknesses to protect the habitat. Afterwards, the truss module calculates the truss mass and critical buckling force for constraint comparison with the thrust. Also, the habitat module calculates the habitat structure and crew supply masses. Finally, the configuration module combines geometries of the reactor, radiators, power conversion section, truss, and crew habitat to determine a shadow angle that shields all subsystems. The configuration module also generates an geometry output file that can be used to plot vehicle designs in Tecplot. Combined with the thickness requirement calculated earlier, the shield volume is now calculated to obtain a shield mass. At this point, all of the system masses are known, and the simulator assesses the constraints for trip time, propellant reserve, and truss force (and one more constraint explained below) to calculate penalty function values. These penalty masses are added to the system mass to obtain the vehicle fitness.

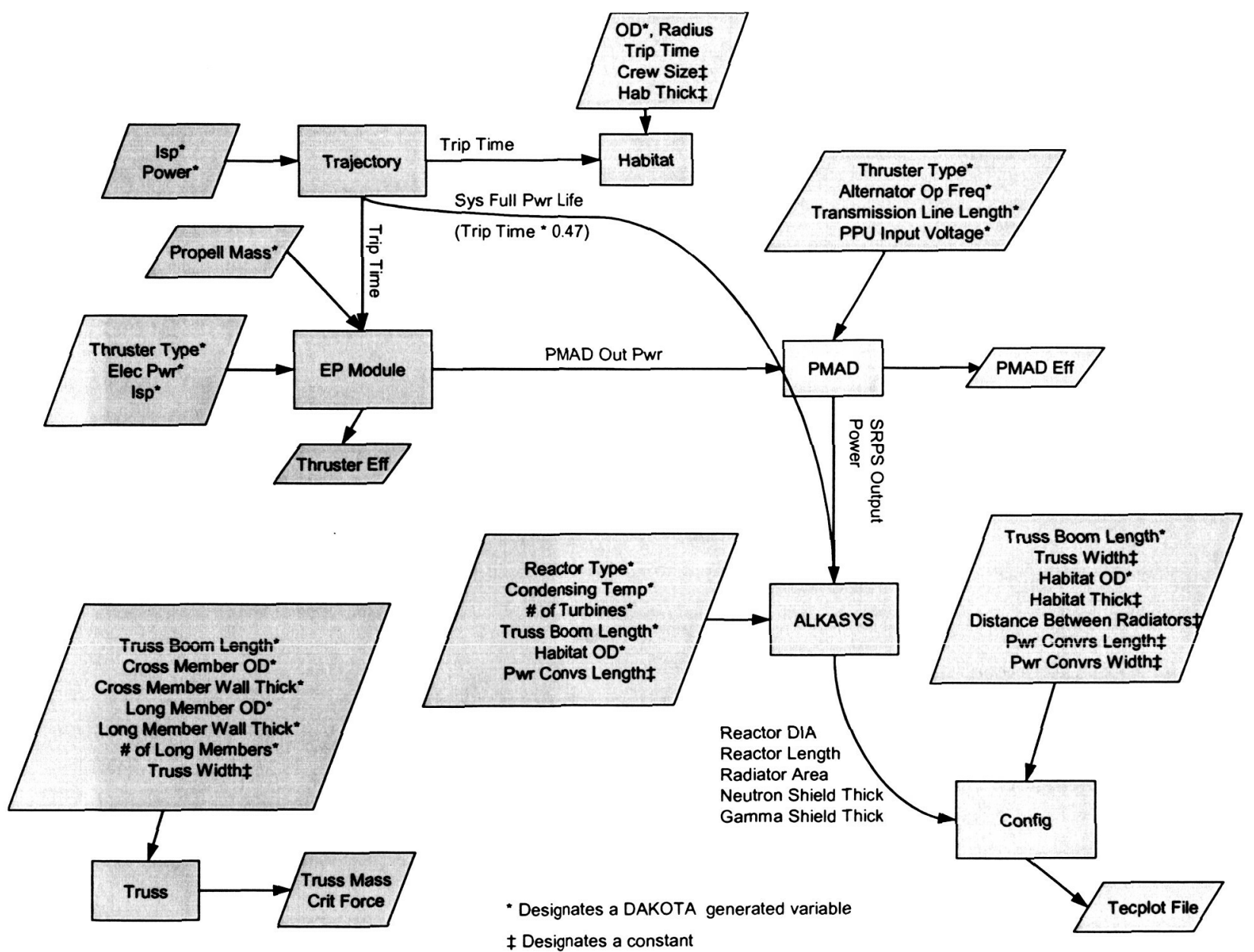

Figure 6. NEVOT flowchart.

Some assumptions have been made due to concerns over computation time as well as limited analysis capability for certain modules. For example, the trajectory code uses a lookup table of VariTOP optimum curves for vehicles with initial mass of $200,000 \mathrm{~kg}$ and specific mass (alpha) of $4 \mathrm{~kg} / \mathrm{kW}$. This has led to an extra penalty function (mentioned above) for specific mass. Unfortunately, there is little trajectory data for low-thrust missions to the asteroids in current literature. Accordingly, the calculations for mission trip time and required propellant mass are approximate, but they allow for the basic trade analyses presented in a later section of this paper. 


\section{Penalty Functions And Scaling Factors}

As mentioned earlier, penalty functions are a means for imposing design constraints on the fitness function in genetic algorithm optimization. NEVOT penalty functions consist of a scale factor and a penalty ratio. The penalty ratio is determined as follows. If the constraint is $x_{\text {design }} \leq x_{\text {constra int }}$, then the penalty ratio ( $\mathrm{p}$ ) is given by

$$
\begin{array}{ll}
p=0 & \text { if } x_{\text {design }} \leq x_{\text {constra int }} \\
p=1-\frac{x_{\text {constra int }}}{x_{\text {design }}} & \text { if } x_{\text {design }}>x_{\text {constra int }}
\end{array}
$$

The penalty ratio is always between zero and one if the design and constraint values are both positive. This ratio must be scaled so that it will have a large enough impact on the fitness function when added to the vehicle mass. If a scale factor is too low, then the fitness function is not significantly affected by constraint violations. The "optimal" design output by the GA may have very low mass but also violate many constraints. On the other hand, if a scale factor is too high the optimization results will be conservative because there will be a large fitness difference between designs that violate constraints and those that do not. The goal is to find a compromise wherein solutions with small constraint violations but low mass are not eliminated immediately. These designs, while not completely feasible, are very close to those designs that meet the constraints at low mass. Thus, their genetic data should be competitive against high-mass designs with all constraints satisfied.

Early in the project it was decided that the design vehicle mass should be used as the penalty scaling factor. Results of the runs showed that the optimal designs were always violating many of the constraints. These designs had such lower mass than some other designs in the same generation that the difference in penalty scaling was too large. Effectively these designs were not assessed as much penalty mass for large constraint violations as those designs with higher mass and small penalty violations. A new scaling factor was needed that would be unbiased within a generation.

A value of $600,000 \mathrm{~kg}$ (a conservative estimate for optimal vehicle mass) was chosen so that conservative designs could be selected to ensure that the mission was possible. When this penalty scaling factor was implemented, the optimal designs often only violated the specific mass (alpha) constraint. It was apparent that a specific mass of $4 \mathrm{~kg} / \mathrm{kW}$ was too ambitious for this manned mission with the available analyses. Since the trajectory assumptions of $200,000 \mathrm{~kg}$ vehicle initial mass and $4 \mathrm{~kg} / \mathrm{kW}$ specific mass could not be met, the trip time scaling factor was decreased so that the trajectory code would have smaller effect on the optimal designs. This showed that scaling factors for separate constraints could be varied to give priority to one constraint over the other. This allowed the code to optimize for mass and specific mass in an attempt to offset the errors in propellant mass calculations by the trajectory code.

\section{Choosing Design Variables for Optimization}

A major challenge in systems design optimization is choosing which parameters to designate as design variables for the code. It is possible to choose every variable on the vehicle, but the resulting computation time would be enormous. Additionally, the purpose of this systems design study is to identify parameters having the greatest impact on system performance and to determine appropriate values for the parameters. There are many parameters that are dependent on other parameters and can therefore be calculated based on engineering analysis. Adding dependent parameters to the variable list also adds the necessity for constraint functions. For instance, the critical buckling force of the truss (a result of several design variables) must be checked against the actual delivered thrust of the electric propulsion device (a result of several other design variables). Adding constraint functions forces the genetic algorithm to explore more constraint values and consume greater computational resources. If the designers can choose to design a dependent parameter to its constraint, then they should implement that logic into their analysis codes.

Initially, five design variables and constraints were utilized in addition to those mentioned in the previous sections. These variables were the shield smaller diameter, gamma shield thickness, neutron shield thickness, shadow shield angle, and radiator area. These values were checked against calculated constraints for each. Inspection of the relationships showed that the shield shadow angle and small diameter could be calculated based on vehicle geometries. The other three variables can be designed to the calculated constraints from the SRPS code. Having a thicker shield than is necessary for a certain truss length only adds waste mass to the vehicle. The result of extra radiator area over the waste rejection requirement is the same. By removing these five variables and their five 
associated constraints, the GA was able to work on fewer variables and constraint functions without loss of quality design space.

Previous versions of the NEVOT software assigned a constant $I_{\mathrm{sp}}$ based on the thruster type that the GA within DAKOTA picked. For example, for each time that DAKOTA picked MPD thrusters, an $I_{\mathrm{sp}}$ of 8000 was assigned. This assumption was acceptable as a first estimate; however, a more robust analysis was desired. Therefore, the code was modified to allow DAKOTA to choose the $I_{\mathrm{sp}}$ from a given range. The most recent versions of NEVOT also included realistic values of thruster efficiency, as opposed to assuming 100 percent efficiency.

Table 1. Selected results from a) NEVU' version with $100 \%$ thruster efficiency, and b) NEVOTT version with realistic thruster efficiencies and variable specific impulse.

\begin{tabular}{|c|c|c|c|c|c|c|c|c|c|c|}
\hline Case & $\begin{array}{l}\text { SRPS } \\
\text { Mass } \\
(\mathrm{kg})\end{array}$ & $\begin{array}{c}\text { Neutron } \\
\text { Shield } \\
\text { Mass } \\
(\mathrm{kg})\end{array}$ & $\begin{array}{c}\text { Radiator } \\
\text { Mass } \\
\text { (kg) }\end{array}$ & $\begin{array}{l}\text { PMAD } \\
\text { Mass } \\
\text { (kg) }\end{array}$ & $\begin{array}{c}\text { Thruster } \\
\text { Type }\end{array}$ & $\begin{array}{l}\text { Propellant } \\
\text { Mass (kg) }\end{array}$ & $\begin{array}{c}\text { Trip } \\
\text { Time } \\
\text { (days) }\end{array}$ & $\begin{array}{c}\text { Jet } \\
\text { Power } \\
(\mathrm{kW})\end{array}$ & $\begin{array}{l}\text { Specific } \\
\text { Mass } \\
\text { (Alpha) } \\
(\mathrm{kg} / \mathrm{kW}) \\
\end{array}$ & $\begin{array}{c}\text { Total } \\
\text { Vehicle } \\
\text { Mass } \\
(\mathrm{kg}) \\
\end{array}$ \\
\hline \multicolumn{11}{|c|}{ a) Thruster Efficiency 100 Percent, Constant $I_{s p}$ for Each Thruster } \\
\hline 1 & 9006 & 775 & 14158 & 9167 & Ion & 96424 & 1631 & 5479 & 21.42 & 249551 \\
\hline 2 & 6836 & 2301 & 10242 & 6865 & Ion & 79031 & 1750 & 3937 & 26.22 & 220148 \\
\hline 4 & 9569 & 986 & 14611 & 9000 & Ion & 98185 & 1626 & 5606 & 22.67 & 260933 \\
\hline 5 & 7154 & 1114 & 10332 & 8100 & MPD & 97397 & 1750 & 4005 & 19.15 & 212991 \\
\hline 8 & 19573 & 1091 & 28200 & 14160 & MPD & 213942 & 1505 & 9627 & 11.08 & 354121 \\
\hline 9 & 19602 & 809 & 29189 & 15875 & MPD & 219388 & 1473 & 10884 & 10.52 & 366769 \\
\hline 11 & 11972 & 1029 & 18010 & 9894 & Ion & 118714 & 1620 & 5805 & 22.23 & 283305 \\
\hline 16 & 12259 & 765 & 15252 & 8199 & MPD & 118040 & 1684 & 4635 & 18.88 & 242276 \\
\hline 17 & 15996 & 831 & 23746 & 13367 & MPD & 197564 & 1522 & 8854 & 11.79 & 335771 \\
\hline 18 & 18536 & 889 & 28066 & 15044 & MPD & 208469 & 1497 & 9987 & 12.04 & 362025 \\
\hline 19 & 20357 & 789 & 30978 & 16736 & MPD & 232885 & 1455 & 11708 & 11.07 & 395126 \\
\hline 21 & 13210 & 1009 & 20381 & 12535 & MPD & 171297 & 1545 & 7747 & 11.97 & 298246 \\
\hline \multicolumn{11}{|c|}{ b) Current Version: Realistic Thruster Efficiency, $I_{\mathrm{sp}}$ Selected by GA } \\
\hline 64 & 28094 & 940 & 41858 & 22097 & MPD & 191038 & 1489 & 11229 & 15.80 & 401712 \\
\hline 68 & 37317 & 1183 & 57988 & 29071 & MPD & 198007 & 1412 & 15475 & 12.87 & 429000 \\
\hline 69 & 32227 & 921 & 50682 & 25818 & MPD & 172355 & 1448 & 13581 & 13.53 & 388549 \\
\hline 70 & 32141 & 1005 & 50529 & 25789 & MPD & 171426 & 1448 & 13567 & 13.60 & 388415 \\
\hline 72 & 36670 & 1528 & 57311 & 28822 & MPD & 191576 & 1416 & 15299 & 12.95 & 421567 \\
\hline 76 & 37452 & 1182 & 58200 & 29430 & MPD & 219127 & 1410 & 15590 & 13.02 & 453862 \\
\hline 77 & 33017 & 881 & 51878 & 26361 & MPD & 177571 & 1441 & 13910 & 13.25 & 394223 \\
\hline
\end{tabular}

\section{Trend Analysis}

With the penalty scaling factors and design variables selected, the code was then executed for trend analysis. It was immediately apparent that thruster efficiency has a large impact on optimal vehicle designs. The reactor, PMAD, radiator, and thruster masses were typically larger when using realistic thruster efficiencies. Also, the jet power was usually higher. As would be expected, these results also translated into greater total vehicle masses. Furthermore, with efficiencies included, MPD thrusters were picked by the GA nearly every time.

It was expected that MPD thrusters would be the best option for high-power manned NEP missions, since the ion thruster masses at the power levels required to complete the mission are enormous compared to those of the MPD thrusters. The better specific power capabilities of the MPD thrusters should enable them to overcome their lower specific impulse as compared to ion thrusters. However, it was also expected that for more extensive trajectory analysis, the propellant mass will increase for all vehicles due to worse specific mass (alpha) values than the current assumption.

Table 1 shows some of the best vehicle designs obtained using the NEVOT codes with (a) thruster efficiency of 100 percent and constant $I_{s p}$ for each thruster, and (b) realistic thruster efficiencies and variable $I_{s p}$ (i.e. $I_{s p}$ selected by the GA). A number of conclusions can be drawn from Table 1. First, it is apparent that the target vehicle specific mass or alpha value of $4 \mathrm{~kg} / \mathrm{kW}_{\mathrm{e}}$ is too ambitious for an NEP mission to the asteroids. The best specific 
mass value achieved for 100 percent thruster efficiency is 10.5 , and for realistic thruster efficiencies the best value is 12.87. Further, as stated previously, the thruster efficiency is seen to have a major effect on the mass of the reactor (SRPS), radiators, PMAD, and propellant. This points out the great need for further development work with electric thrusters. Further observations from Table 1 regarding thrusters are that (1) ion thrusters typically result in lower vehicle mass, but higher vehicle alpha and longer trip times, and (2) MPD thrusters generally provide the best trip times and alpha values, but they need more propellant and result in heavier vehicles.

Since the trade results accounting for thruster efficiency are generally more realistic for assessing NEP, the remainder of this section will focus on those results. Further results for the case of 100 percent thruster efficiency are given in Ref. 10.

It quickly became clear in these studies that vehicle specific mass (alpha) has a large (and approximately linear) impact on fitness, which is shown in Figure 7. This was expected, because a large mass penalty is imposed upon vehicle designs that fail to meet the alpha fitness constraint. However, alpha and total vehicle mass have no obvious correlation, which is shown in Figure 8.

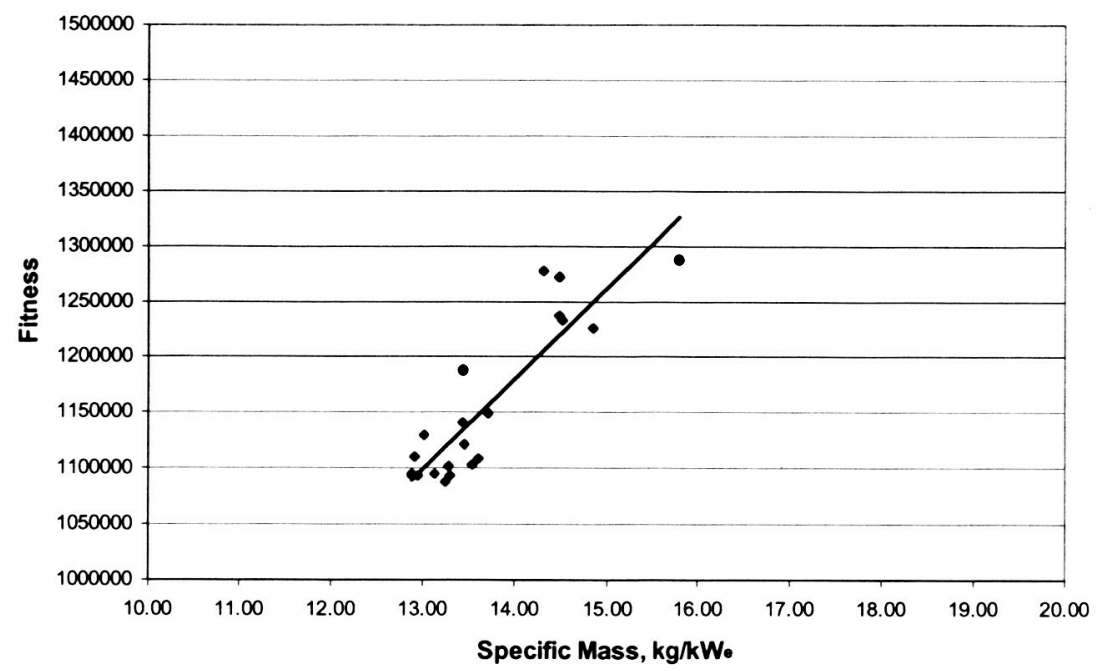

Figure 7. Fitness increase due to increases in specific mass.

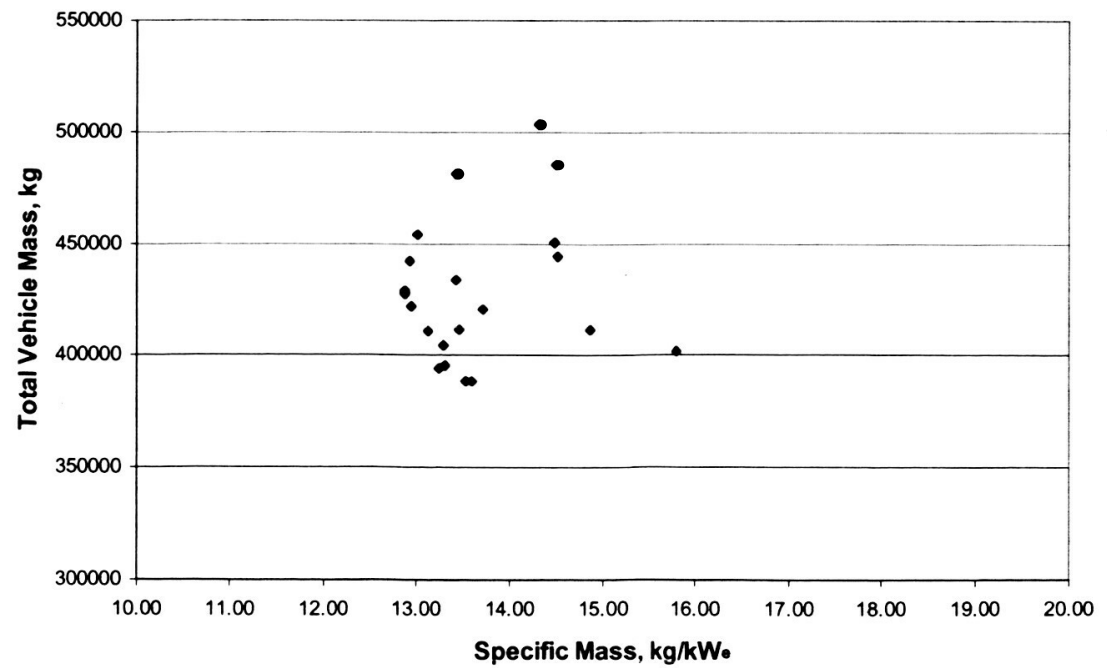

Figure 8. Specific mass versus total vehicle mass. 
Other important trade studies show the effect of vehicle (truss) length on the shield mass and total vehicle mass. As the truss length increases, the mass of the radiation shield decreases. This result is shown in Fig. 9, and was expected, because the crew habitat would experience a lower radiation dose with increasing distance from the reactor. This lower shield mass outweighs the increase in truss mass required by the longer truss. Figure 10 shows how vehicle mass decreases slightly with longer truss length.

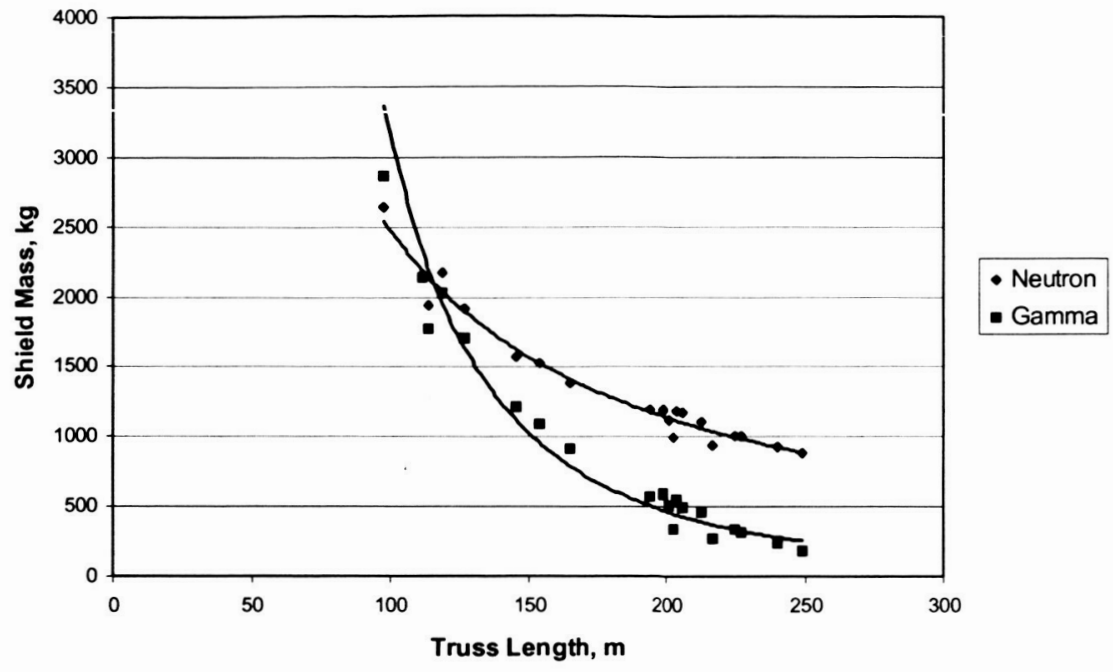

Figure 9. Radiation shield mass variation with truss length.

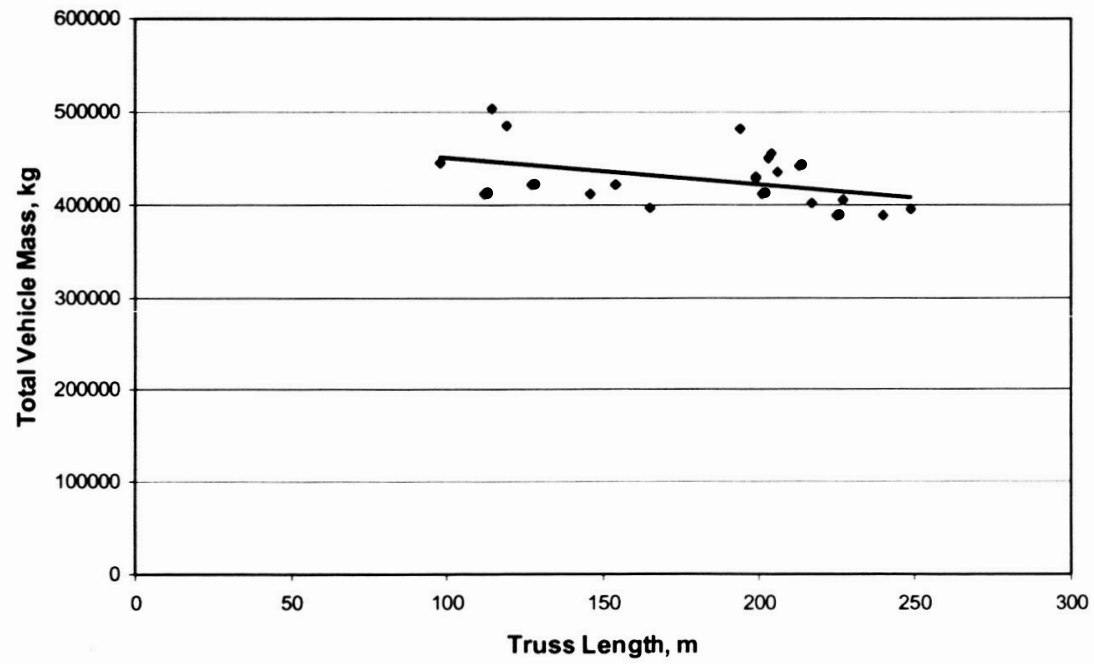

Figure 10. Total vehicle mass variation with truss length.

Thruster jet power also obviously plays a pivotal role in vehicle performance. Trend analysis dramatically showed that as the jet power increases, the trip time decreases (Fig. 11). However, Fig. 12 shows how the total vehicle mass increases with jet power. This means that higher jet power carries a price to be paid in vehicle mass, specifically mass of the propellant, reactor, radiators, and PMAD, as shown in Table 1 . The dependencies shown in Figure 11 and Figure 12 were expected, yet the results illustrate the utility and value of the NEVOT optimization tool for trade studies. 


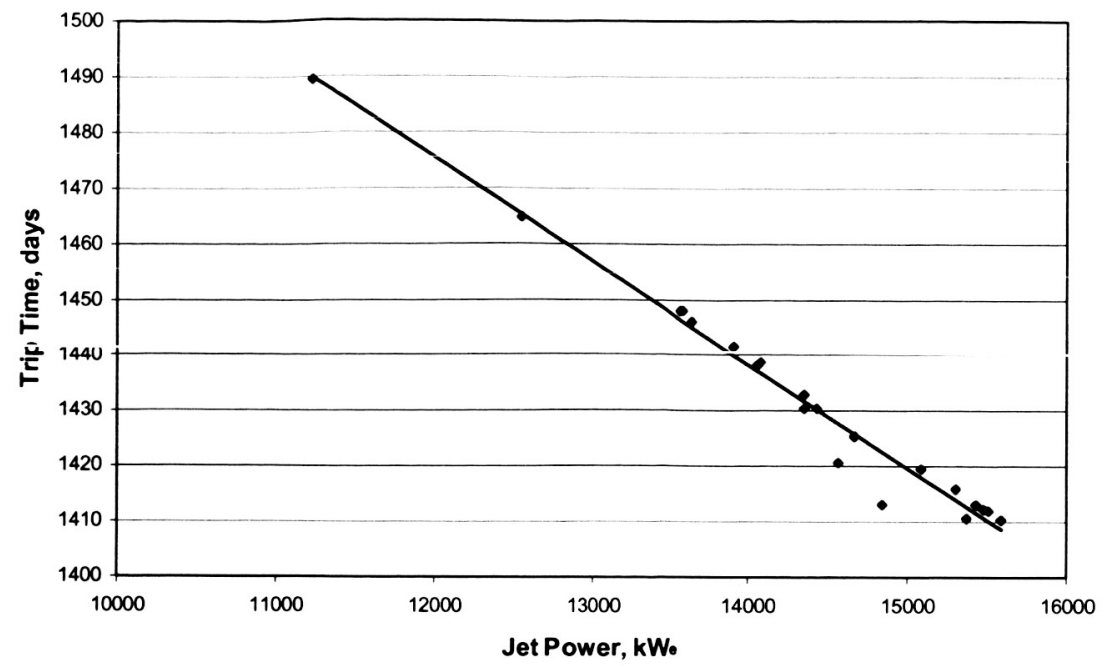

Figure 11. Trip time reduction with increased jet power.

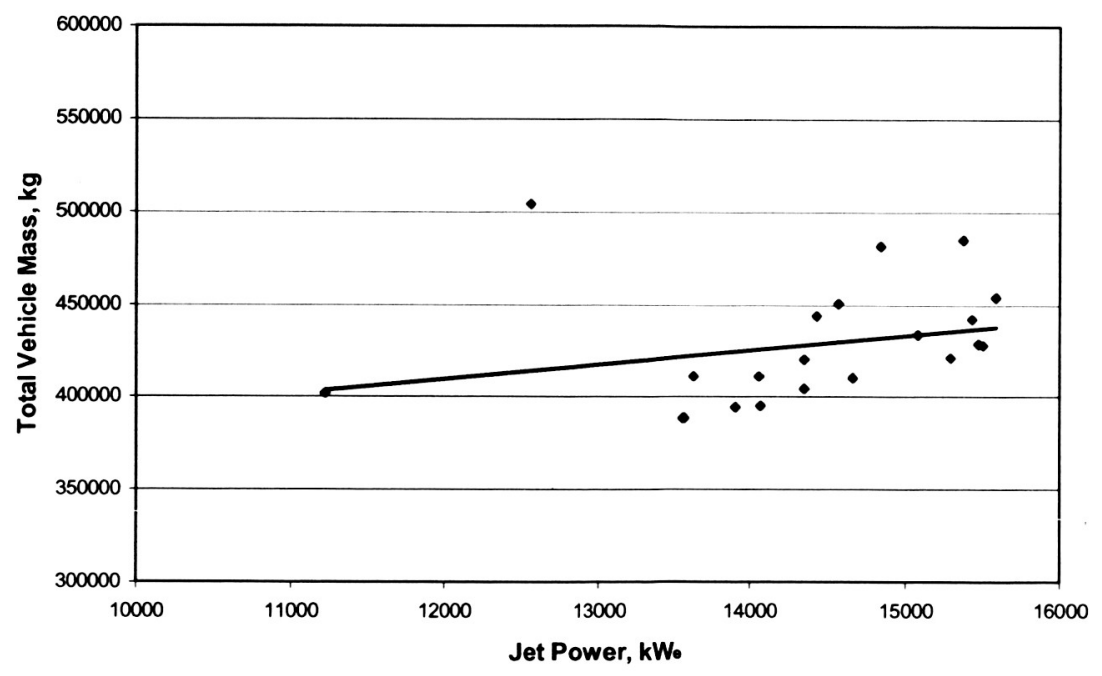

Figure 12. Vehicle mass increase with increased jet power.

\section{Optimal Vehicle Configurations}

Genetic algorithm-based optimization yields a group of most promising vehicle designs in a global sense. Stated differently, optimization of complex systems such as space vehicles with GA's does not provide one best design, but rather a set of designs that must be then investigated in more detail by the engineering design team. This occurs due to the complexity of the optimization problem and the large number of design variables. For example, if the top ten vehicles obtained in GA-based optimization were examined closely, many of them would be similar visually, but some of the subsystems would have significant differences. For this reason, the discipline experts on the design team must carefully review several of the highest performing systems to determine which are truly feasible for development.

Figure 13 shows the best NEP vehicle configurations determined using NEVOT for the version that uses 100 percent thruster efficiency and constant $I_{\mathrm{sp}}$ for each thruster type and Fig. 14 shows the best vehicle configuration using the current version with realist thruster efficiency and $I_{\mathrm{sp}}$ chosen by the GA. Figures 16 through 18 show the 
next 4 best NEP vehicle configurations with the new NEVOT version that uses realistic thruster efficiency and $I_{s p}$ selected by the GA.

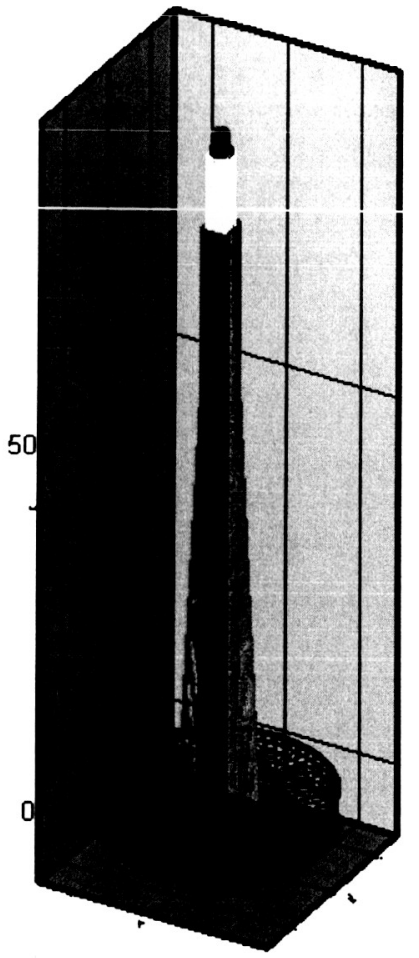

Figure 14. Top vehicle configuration using version with $100 \%$ thruster efficiency and constant $I_{\text {sp. }}$

Fitness $=693601$

Vehicle Mass $=211991 \mathrm{~kg}$

Jet Power $=4005 \mathrm{~kW}_{\mathrm{e}}$

Specific Mass $=19.15 \mathrm{~kg} / \mathrm{kW}_{\mathrm{e}}$

Trip Time $=1750$ days

SRPS Mass $=7154 \mathrm{~kg}$

Radiator Mass $=10332 \mathrm{~kg}$

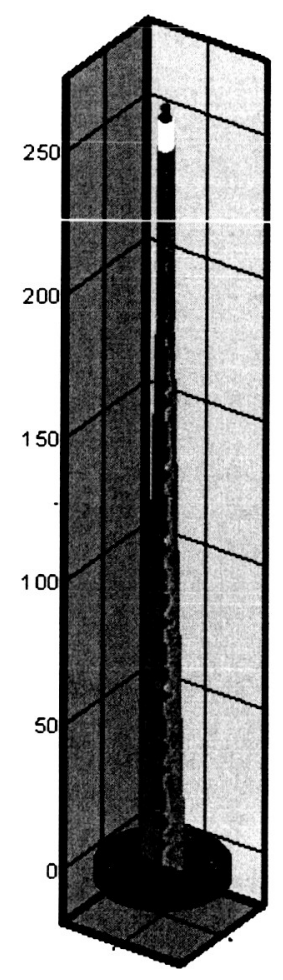

Figure 14. Best vehicle configuration using current version with realistic thruster efficiency and variable $I_{\text {sp. }}$.

Fitness $=1087970$

Vehicle Mass $=394223$

Jet Power $=13910 \mathrm{~kW}_{\mathrm{e}}$

Specific Mass $=13.25 \mathrm{~kg} / \mathrm{kW}_{\mathrm{e}}$

Trip Time $=1441$ days

SRPS Mass $=33017 \mathrm{~kg}$

Radiator Mass $=51878 \mathrm{~kg}$ 


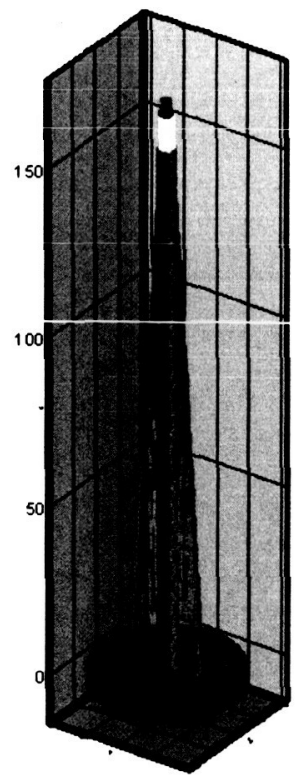

Figure 16. Second best vehicle using current version.

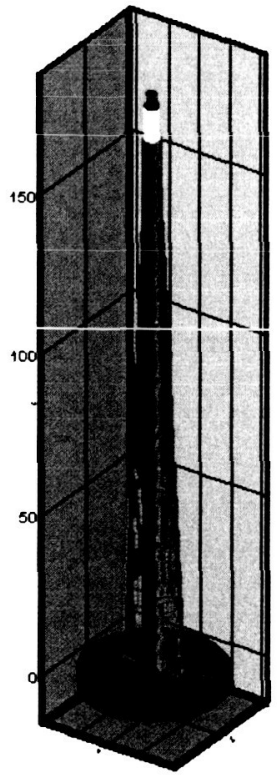

Figure 17. Third vehicle using current
Fitness $=1093043$

Vehicle Mass $=395299 \mathrm{~kg}$

Jet Power $=14074 \mathrm{~kW}$

Specific Mass $=13.30 \mathrm{~kg} / \mathrm{kW}_{\mathrm{e}}$

Trip Time $=1439$ days

SRPS Mass $=33448 \mathrm{~kg}$

Radiator Mass $=52532 \mathrm{~kg}$

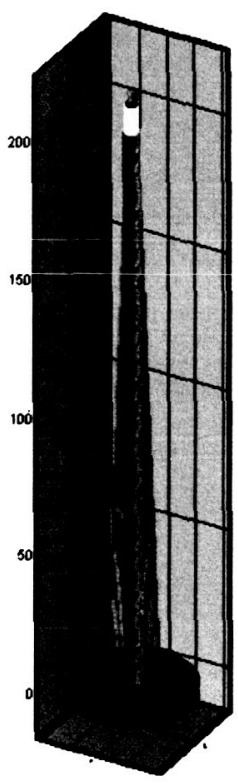

Fitness $=1093552$

Vehicle Mass $=427742 \mathrm{~kg}$

Jet Power $=15506 \mathrm{~kW}_{\mathrm{e}}$

Specific Mass $=12.88 \mathrm{~kg} / \mathrm{kW}_{\mathrm{e}}$

Trip Time $=1412$ days

SRPS Mass $=37237 \mathrm{~kg}$

Radiator Mass $=57852 \mathrm{~kg}$

Figure 18. Fourth best vehicle using current version.

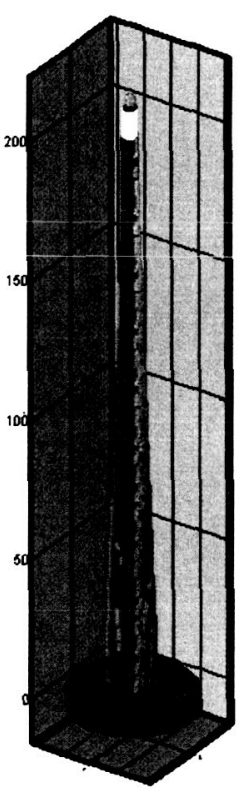

Fitness $=1094284$

Vehicle Mass $=429000 \mathrm{~kg}$ Jet Power $=15475 \mathrm{~kW}_{\mathrm{e}}$ Specific Mass $=12.87 \mathrm{~kg} / \mathrm{kW}_{\mathrm{e}}$ Trip Time $=1412$ days SRPS Mass $=37317 \mathrm{~kg}$ Radiator Mass $=57988 \mathrm{~kg}$

Figure 19. Fifth best vehicle using current version. 


\section{Summary and Conclusions}

By combining subsystem analysis codes with genetic algorithm-based optimization, NEVOT provides a useful tool for conceptual design studies of a nuclear electric propulsion vehicle. The modular nature of the subsystem codes allows for modifications to be made easily when better analysis data becomes available. Also, the framework is in place for other systems designs studies such as surface space nuclear power missions. Trade studies based on the optimal designs from a series of NEVOT runs demonstrated that the thruster type and thruster efficiency have a major effect on NEP vehicle performance. The implication of these findings is that considerable effort should be devoted to research and development of electric thrusters for future exploration missions. Vehicles with MPD thrusters show the most promise for manned NEP missions due to their high power capability with relatively low

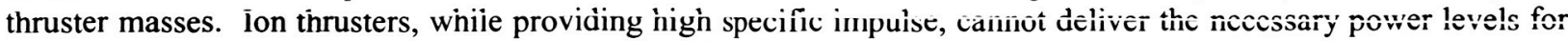
the reference mission to the asteroids without requiring large clusters of thrusters.

Results also showed the effect of jet power on vehicle performance (reduced trip time), and that increases in jet power must be paid for through mass increases for the propellant, reactor, radiators, and PMAD. Finally, it was interesting to note that the vehicle length or truss length had a major impact on the reactor shield mass. That is, the longer the vehicle, the thinner and lighter the reactor shield can be to provide the necessary radiation protection for the crew. As a result, vehicle mass actually decreased slightly for increased truss length.

\section{References}

${ }^{1}$ Smith, B. K., "Definition, Expansion, and Screening of Architectures for Planetary Exploration Class Nuclear Electric Propulsion and Power Systems," Masters Thesis, Massachusetts Institute of Technology, Cambridge, MA, 2003.

${ }^{2}$ Ananthasuresh, G.K., Kota, S. and Gianchandani, Y., 1994, "A Methodical Approach to the Design of Compliant Micromechanisms," Solid-State Sensor and Actuator Workshop, Hilton Head Island, South Carolina, pp. 189-192

${ }^{3}$ Kota, S., 1999, "Design of Compliant Mechanisms. Applications to MEMS," SPIE Conference on Smart Electronics and MEMS, Newport Beach, California, Vol. 3673, pp. 45-54.

${ }^{4}$ Parkinson, M. Howell, L. and Cox, J., "A parametric approach to the optimization based design of compliant mechanisms," Proceedings of the ASME 1997 Design Engineering Technical Conferences, DETC97/MECH-3763.

${ }^{5}$ Roberts, F S., 1976 "Discrete Mathematical Models with Applications to Social, Biological and Environmental Problems", Prentice-Hall, Phoenix, Az.

${ }^{6}$ Parsons, R. and Canfield, S. 2002 "Developing Genetic Programming Techniques for the Design of Compliant Mechanisms", Journal of the International Society for Structural and Multidisciplinary Optimization, 24(1), pp. 7886.

${ }^{7}$ Deb, K., Pratap, A., Agarwal, S., and Meyarivan, T., "A fast and elitist multiobjective genetic algorithm: NSGA-II" IEEE Transactions on Evolutionary Computation, Vol. 6, No. 2., Apr 2002.

${ }^{8}$ Chapman, C. D., Saitou, K. and Jakiela, M. J., 1994, "Genetic Algorithms as an Approach to Configuration and Topology Design”, Journal of Mechanical Design, 116, pp. 1005-1012.

${ }^{9}$ Tinker, M. L., Steincamp, J. W., Stewart, E. T., Patton, B. W., Pannell, W. P., Newby, R. L., Coffman, M. E., Qualls, A. L., Greene, S., Bancroft, S., and Molvik, G., "Nuclear Electric Vehicle Optimization Toolset (NEVOT)," AIAA-2004-4552, Proceedings of $10^{\text {th }}$ AIAA/ISSMO Multidisciplinary Analysis and Optimization Conference, Aug. 29-Sept. 1, 2004, Albany, NY.

10 Irwin, R. W., and Tinker, M. L., "Preliminary Design of a Manned Nuclear Electric Propulsion Vehicle Using Genetic Algorithms," in proceedings of Space Technology and Applications International Forum (STAIF) 2005, Feb. 13-17, 2005, Albuquerque, NM. 